\title{
La afiliación a la seguridad social de los trabajadores y sus efectos en las finanzas de las empresas en México
}

\author{
Carlos Mauricio Tosca-Vidal* \\ Germán Martínez-Prats** \\ Candelaria Guzmán-Fernández ${ }^{* * *}$
}

Fecha de recepción: 15 de diciembre de 2020

Fecha de aprobación: 4 de mayo de 2021

Resumen: El objetivo de este artículo es determinar las consecuencias de la afiliación de los trabajadores al sistema de seguridad social con un salario inferior al real en México. Existe poca literatura acerca de la afectación de estas malas prácticas. Se realizó una investigación descriptiva tanto desde el punto de vista legal -en lo relativo a las sanciones-, como financiero - en cuanto afecta a los dueños de las organizaciones-. Tiene un enfoque cualitativo y una técnica documental. De la investigación se determinó que existen múltiples afectados: Estado, trabajadores y patrón. El Estado, porque deja de percibir el $43 \%$ de las cuotas; el trabajador, porque al tener un accidente o incapacidades, el Estado, a través del Instituto de Seguridad Social, le reconoce sus prestaciones según el salario con el que esté inscrito; y el patrón, pues si el trabajador se accidenta, tiene la obligación de pagarle las prestaciones faltantes, para lo cual debe formar un capital constitutivo. Se concluye que los patrones deben tener en cuenta todas las consecuencias que acarrea disminuir el salario de los trabajadores al inscribirlo en la seguridad social, debido a que ponen en riesgo las finanzas de la organización y pueden llegar a ser sancionados con privación de la libertad, por dejar en estado de indefensión al trabajador.

Palabras clave: trabajador, salario, finanzas, patrón, seguridad social, Estado.

Clasificación JEL: E26, E27, H26, J01, J28

Cómo citar:

Tosca Vidal, C. M., Martínez Prats, G., \& Guzmán Fernández, C. (2021). La afiliación a la seguridad social de los trabajadores y sus efectos en las finanzas de las empresas en México. Apuntes del Cenes, 40(72). Págs. 71 - 86. https://doi.org/10.19053/01203053.v40.n72.2021.12246

\footnotetext{
* Candidato a doctor en Estudios Económico-Administrativos en la Universidad Juárez Autónoma de Tabasco. México. tosca_vidal@ hotmail.com (iD https://orcid.org/0000-0002-1546-8832

** Doctor en Gobierno y Administración Pública. Profesor investigador de tiempo completo en la Universidad Juárez Autónoma de Tabasco en la División de Ciencias Económico-Administrativas. México germanmtzprats@hotmail.com (iD https://orcid.org/0000-00016371-448X Contacto de correspondencia.

*** Doctor en Gestión Estratégica y Políticas de Desarrollo. Profesor investigador de tiempo completo de la Universidad Juárez Autónoma de Tabasco en la División de Ciencias Económico-Administrativas. candyguzmanf@hotmail.com iD https://orcid. org/0000-0002-6144-7397
} 


\title{
Affiliation to Social Security of Employees and its Effects on the Finances of Companies in Mexico
}

\begin{abstract}
The objective of this paper is to determine the consequences of the affiliation of employees to the social security system with a lower salary than the real one in Mexico. There is little literature about the effect of these bad practices. A descriptive investigation was carried out both from the legal -as regard sanctions-, and financial point of view -as it affects the owners of the organizations. It has a qualitative approach and a documentary technique. From the investigation it was determined that there are multiple affected: State, employees and employer. The State, because it stops receiving $43 \%$ of the Social Security contributions; the employee, because upon having an accident or disability, the State through the Social Security Institute grants his benefits according to the salary with which he is registered; and the employer, because if the employee is injured, that one has the obligation to pay the missing benefits, for which this employer must form a constituent capital. It is concluded that employers must take into account all the consequences of reducing employees' wages when registering them in social security, because they put the finances of the organization at risk and can be sentenced to deprivation of liberty (e.g. imprisonment) for leaving the employee defenseless.
\end{abstract}

Keywords: employee, wage, finance, employer, social security, State. 


\section{INTRODUCCIÓN}

Según el Banco Mundial (2021), en el 2019 la población total del mundo fue de 7674 miles de millones de habitantes. En el ámbito laboral, la población económicamente activa (PEA) -la cual está integrada por todas las personas de 12 años o más que tienen una actividad económica o buscaron realizar una-, representa a nivel mundial 3468 miles de millones de posibles trabajadores.

En México, la PEA, de acuerdo con el Banco de México, en el 2020 era de 53 978 millones de personas. El Instituto Mexicano del Seguro Social (IMSS, 2021) en ese año tenía un registro de afiliados -permanentes y eventualesde 19702 millones de personas, que representan el $36.50 \%$ de la PEA.

Teniendo en cuenta las cifras mencionadas, se observa que más del $50 \%$ de la PEA no tiene servicios de seguridad social, la cual es definida por la Organización Internacional del Trabajo (2021, par. 1) como "[...] la protección que una sociedad proporciona a los individuos y hogares el acceso a la asis- tencia médica y garantizar la seguridad del ingreso, en situaciones de vejez, desempleo, enfermedad, invalidez, accidentes de trabajo, maternidad o pérdida del sostén familiar".

La seguridad social es uno de los derechos fundamentales del hombre. Vale recordar que la Declaración Universal de los Derechos Humanos, adoptada por la Organización de las Naciones Unidas en el año 1948, en el artículo 22 menciona que toda persona tiene derecho, como miembro de la sociedad, a la seguridad social. Pero existe una gran problemática en las naciones para hacer efectivo ese derecho, pues la Organización Internacional del Trabajo (OIT, 2010) publicó que solo una de cada cinco personas cuenta con una cobertura adecuada de seguridad social, situación que evidencia la importancia del tema.

El IMSS anota que la seguridad social mexicana tiene dos normas importantes. La Ley de Accidentes de Trabajo del Estado de México de 1904, promulgada en la época porfiriana, y la Ley de Accidentes de Trabajo del Estado de 
Nuevo León, expedida en 1906. Estas leyes hacen parte de la primera normativa de la historia donde se consagraba la obligación de los empresarios de proporcionar a sus empleados atención médica en caso de enfermedad, accidente o muerte, con ocasión del cumplimiento de sus labores. En 1915 se sancionó la Ley de Accidentes, que establecía las pensiones e indemnizaciones a cargo del empleador, en caso de incapacidad o muerte del trabajador a causa del cumplimiento de su trabajo.

En 1917 en la Constitución Política de los Estados Unidos Mexicanos (2019) se reconoce la seguridad social en el artículo 123, apartado A en la F. XXIX. La Ley del Seguro Social es de utilidad pública y comprende seguros de invalidez, de vejez, de vida, de cesación involuntaria del trabajo.

En el sexenio del presidente Lázaro Cárdenas se presentaron anteproyectos de la Ley del IMSS; el 2 de junio de 1941 se instaura la comisión técnica redactora de la Ley del Seguro Social (LSS), el 31 de diciembre de 1942 se expide la LSS y entra en vigor el 1 de enero de 1943.

El sistema de seguridad social en el país está a cargo de entidades o dependencias públicas, federales o locales y de organismos descentralizados mediante los cuales se busca garantizar el derecho a la salud, a la asistencia médica, la protección de los medios de subsistencia y los servicios sociales necesarios para el bienestar individual y colectivo.

Las entidades con mayor número de población beneficiada son el IMSS y el Instituto de Seguridad de Servicios Sociales de los Trabajadores del Estado (ISSSTE).

\section{El Centro de Estudios de las Finanzas} Públicas (2013) publicó que, para finales del 2012, seis de cada diez personas ocupadas en el país no contaban con acceso a instituciones de salud. Esto significa que el $60 \%$ de las personas que se encuentran en el mercado laboral no están registradas en el IMSS o en el ISSSTE.

El artículo 2 de la LSS indica que la seguridad social tiene por finalidad garantizar el derecho a la salud, la asistencia médica, la protección de los medios de subsistencia y los servicios sociales necesarios para el bienestar individual y colectivo, así como el otorgamiento de una pensión que, en su caso y previo cumplimiento de los requisitos legales, será garantizada por el Estado. Los ingresos del IMSS serán aportados por el gobierno, patrones y trabajadores.

El cálculo y la emisión de la liquidación de las cuotas obrero-patronales eran responsabilidad del IMSS, pero a partir de la reforma de 1993 son los patrones los que deben determinar la liquidación pertinente. 
Basados en la experiencia de los autores de este trabajo se puede afirmar que los patrones consideran que la aportación al IMSS es excesiva; en la actualidad la cuota obrera al Instituto, para salarios que no excedan de tres salarios mínimos generales, aporta el $2.775 \%$-ramas enfermedades y maternidad (EM), invalidez y vida (IV), cesantía en edad avanzada o vejez (CV) - y la cuota patronal en las mismas ramas es del $7.75 \%$.

Para hacer algunos breves comentarios sobre los diferentes sistemas de seguridad social en América Latina, se toman como referencia cinco países: Colombia, Brasil, México, Chile y Costa Rica.

En Brasil, la seguridad social se proporciona a través del Sistema Único de Salud (SUS), que cubre alrededor del $75 \%$ de la población, obtiene su financiamiento con impuestos generales y contribuciones que se recaudan en los diferentes niveles de Gobierno (Montekio et al., 2011).

En Chile, para diciembre de 2020, el sistema público de salud cubría el $68.90 \%$ de la población ocupada. En este país los encargados de toda la seguridad social son el Ministerio de Salud y los organismos dependientes: para el sector público es FONASA y para el sector privado es el ISAPRE, los cuales conforman el Sistema Nacional de Servicios de Salud (SNSS) (Subsecretaría de Previsión Social, 2021).
En Colombia, el Sistema General de Seguridad Social en Salud (SGSSS) se compone de dos regímenes: el contributivo (RC), que para el 2020 se estima que existen 23362000 de personas afiliadas; y el subsidiado (RS), que para el mismo año son alrededor de 24027 000. En 1995 el 29.21 \% de la población estaba afiliada a algún tipo de régimen y en 2020 se encuentra afiliado el $97.78 \%$ de la población nacional (Ministerio de Salud y Protección Social [Minsalud], 2021).

En Costa Rica, los servicios de salud públicos le corresponden a la Caja Costarricense de Seguro Social (CCSS), institución autónoma que administra tres regímenes: el seguro de enfermedad y maternidad, el seguro de invalidez, vejez y muerte, y el régimen no contributivo. Obtiene financiamiento de los afiliados, los empleadores y el Estado (Sáenz et al., 2011).

En México, en el sector público comprende la seguridad social que cubre a los trabajadores del sector formal a través de entidades como el IMSS, ISSSTE, PEMEX, entre otros, y las entidades que prestan servicios a la población sin seguridad social como el seguro del bienestar. Los servicios del IMSS, ISSSTE se financian de manera tripartita: trabajador, patrón y Estado. En cambio, en el seguro del bienestar, la Secretaría de Salud y los servicios estatales de salud se financian por el Gobierno federal y por 
algunas cuotas de recuperación que pagan los usuarios al recibir el servicio (Montekio et al., 2011).

Los patrones consideran que la aportación al IMSS es excesiva, pues en la actualidad la cuota obrera al IMSS para salarios que no exceden de tres salarios mínimos generales es de $2.775 \%$ (ramas: EM, IV, CV) y la cuota patronal en las mismas ramas es de $7.75 \%$. Los porcentajes indican que sí hay una diferencia importante en lo que aporta el patrón.

Es importante anotar que, además de las cuotas señaladas, el patrón aporta:

- $20.40 \%$ de cuota fija

- $1 \%$ de guardería

- $2 \%$ retiro

- $5 \%$ INFONAVIT

- Del $0.50 \%$ hasta el $15 \%$ según la actividad por RT.

De acuerdo con los datos mencionados, no todos los sujetos obligados cumplen con estos porcentajes, o realizan prácticas deliberadas de evasión o interrupción en el registro. Levy (2010) advierte que algunas de las estrategias de evasión son registrar solo una parte de los trabajadores, rotarlos temporalmente o registrarlos con un salario inferior.

La evasión fiscal afecta a toda la nación, debido a que perjudica las finanzas estatales y limita los recursos del Gobierno para aplicar las políticas. En el contexto del IMSS, el patrón da de alta a sus trabajadores con un salario inferior o, peor aún, no informa que tiene trabajadores que no se encuentran registrados en el IMSS, y por eso tiene el deber legal de pagar las aportaciones correspondientes (Medina \& Flores, 2016).

En el glosario del "Informe tributario y de gestión" emitido por el Servicio de Administración Tributaria (2018, p. 4), se define la evasión fiscal "como toda acción u omisión parcial o total, tendiente a eludir, reducir o retardar el cumplimiento de la obligación tributaria".

Algunas formas de evasión son las siguientes:

1. El patrón se afilia al IMSS y reporta un número inferior de trabajadores del que realmente tiene en su nómina, por lo cual se ahorra la cotización mensual de algunos de ellos, pero se expone a un riesgo innecesario por no pagar lo que realmente le corresponde.

2. El patrón no tiene la prima de riesgo de trabajo de acuerdo con la actividad que desempeña, lo cual significa que no está ofreciendo la cobertura de riesgo que le corresponde a sus empleados.

3. El patrón reporta el número total de trabajadores con un salario inferior al que devengan; en consecuencia, 
el monto mensual de su cotización está por debajo del real que debe pagar al IMSS.

Cortés et al. (2011) mencionan algunas de las causas que los patrones señalan para justificar la evasión de las cuotas de seguridad social:

1. El sentimiento de injusticia ante el sistema tributario.

2. La percepción de bajo riesgo de ser detectado o de la baja severidad del castigo.

3. La percepción de la mala utilización de los recursos públicos y del alto nivel de corrupción administrativa existente.

4. La magnitud de la carga tributaria.

5. El futuro incierto del sistema de pensiones.

6. La alta dispersión de pequeñas empresas y microempresas en el universo productivo.

7. Los altos niveles de informalidad existentes en el país.

8. Los altos niveles de rotación de los trabajadores entre segmentos del mercado de trabajo (formal/informal).

9. Percepción de una falta de eficiencia, eficacia y oportunidad en el acceso a los servicios de salud.

La falta de cultura de pago correcto y las prácticas para evitar la declaración y el pago generan consecuencias sociales y económicas considerables dentro del sistema, como el incumplimiento de la finalidad de buscar el bienestar de las personas, lo cual ocasiona un problema social y una violación a los principios de seguridad social; asimismo causa la disminución de los ingresos al Instituto, la distorsión de la asignación de los recursos, el desplazamiento de la carga tributaria hacia los que declaran correctamente y el aumento de los costos de fiscalización.

Para los trabajadores, las consecuencias representan un grave riesgo, debido a que no podrán contar con las prestaciones económicas reales y su vejez será afectada (Medina \& Flores, 2017)

Cuando el patrón no registra al trabajador con el salario real ante el IMSS, la responsabilidad patrimonial y legal es de él, incluso pone en riesgo su permanencia en el mercado, puesto que la ley le asigna la responsabilidad de asumir las citadas prestaciones en caso de que se presente un accidente de trabajo o una enfermedad.

El artículo 304 de la LSS determina que cuando los patrones y sujetos obligados incurran en actos $u$ omisiones que impliquen el incumplimiento del pago de los conceptos fiscales que señala el artículo 287, deberán asumir el pago de:

- Las cuotas

- Los capitales constitutivos

- Las actualizaciones

- Los recargos

- Las multas impuestas en los términos de ley 
- Los gastos realizados por el Instituto.

En México, todo ordenamiento jurídico tiene sustento en la Constitución Política de los Estados Unidos Mexicanos (CPEUM 123, Fracción XIV), donde se establece lo siguiente:

Los empresarios serán responsables de los accidentes del trabajo y de las enfermedades profesionales de los trabajadores, sufridas con motivo o en ejercicio de la profesión o trabajo que ejecuten; por lo tanto, los patronos deberán pagar la indemnización correspondiente, según haya traído como consecuencia la muerte o simplemente incapacidad temporal o permanente para trabajar, de acuerdo con lo que las leyes determinen. Esta responsabilidad subsistirá aún en el caso de que el patrón contrate el trabajo por un intermediario. (CPEUM 123, Fracción XIV)

En la LFT, en el artículo 472 título noveno (Ley Federal del Trabajo, 2006), se establecen las directrices relativas a los riesgos de trabajo en las relaciones laborales.

Los patrones que inscriben a sus trabajadores en el régimen obligatorio del seguro social quedan relevados del cumplimiento de las obligaciones relacionadas con los riesgos de trabajo que establece la LFT, de acuerdo con el artículo 53 de la LSS.

La CPEUM, la LFT y la LSS regulan las obligaciones de los patrones respecto a los accidentes de trabajo y enfermedades profesionales que sufran los subordinados. Para hacer frente a esa responsabilidad, el IMSS será el encargado de cubrir las prestaciones en especie y dinero, en la medida que el patrón cumpla con las obligaciones de registrar y aportar sus cuotas de manera correcta.

La Comisión Representativa ante Organismos de Seguridad Social (CROSS, 2012) menciona que el capital constitutivo es

el monto pagado por el patrón al Instituto Mexicano del Seguro Social para restituir las erogaciones de las prestaciones económicas y en especie otorgadas por este a un derechohabiente, por un incumplimiento de obligaciones elementales del patrón, tales como: inscribir a los trabajadores, informar en tiempo y forma el salario base de cotización. (CROSS, 2012)

\section{METODOLOGÍA}

La presente investigación es de tipo inductivo, con enfoque cualitativo. Se emplea la técnica documental informativa para analizar la información y generar conclusiones del tema de investigación. Se apoya en el estudio de un caso para ejemplificar el impacto que tienen estas prácticas en las finanzas de las empresas (Hernández \& Mendoza, 2018).

La metodología utilizada en la investigación es la adecuada, debido a que se maneja el estudio de caso de un 
trabajador que fue inscrito en el IMSS con un salario menor que el efectivamente percibido. Se busca presentar un ejemplo que muestre las consecuencias que se acarrean para la empresa, las personas y el IMSS.

\section{RESULTADOS}

Para demostrar el impacto que tiene para las finanzas del IMSS, se realiza un ejercicio en el que se compara el pago de las COP que debió pagar el patrón, es decir, con un salario diario de 238.89 y con el SDI de $\$ 250.00$ (pesos mexica- nos), en el cual se observa que tiene que efectuar un pago total de \$1614.69. En la Tabla 2 se aprecia la integración de los COP que pagó con un SDI de $\$ 100.00$ (60\% menos que el real) con una cuota de $\$ 945.44$, y se tiene como resultado $\$ 669.25$ de evasión de las cuotas, es decir, el daño a las finanzas del IMSS es de más de $\$ 8000.00$ al año, con esos $\$ 945.44$ tiene que darle servicio médico al trabajador y sus dependientes económicos, otorgar servicios de guardería y aportar a su jubilación, lo cual afecta la operatividad del IMSS.

Tabla 1. Cálculo de las COP con salario real

\begin{tabular}{|c|c|c|c|c|c|}
\hline \multicolumn{6}{|l|}{ Cálculo salario real percibido } \\
\hline Salario percibido & \multicolumn{2}{|r|}{$\$ 238.89$} & & & \\
\hline Factor de integración & \multicolumn{2}{|r|}{1.0466} & & & \\
\hline SDI & \multicolumn{2}{|r|}{$\$ 250$} & & & \\
\hline SBC & \multicolumn{2}{|r|}{$\$ 7,600$} & & & \\
\hline Años de antigüedad & \multicolumn{2}{|r|}{2} & & & \\
\hline Cálculo & \multicolumn{2}{|r|}{ Mensual } & & & \\
\hline SMDF 2016 & \multicolumn{2}{|c|}{73.04} & & & \\
\hline Pago IMSS & \multicolumn{2}{|c|}{ Patrón } & \multicolumn{2}{|c|}{ Trabajador } & Total \\
\hline Enfermedades y maternidad C. F. & $20.40 \%$ & 452.96 & & 452.96 \\
\hline Enfermedades y maternidad Excedente & $1.10 \%$ & 10.33 & $0.40 \%$ & 3.76 & 14.08 \\
\hline Enfermedades y maternidad esp. & $0.70 \%$ & 53.20 & $0.25 \%$ & 19.00 & 72.20 \\
\hline E y M esp. Pensionados & $1.05 \%$ & 79.80 & $0.38 \%$ & 28.50 & 108.30 \\
\hline Riesgo de trabajo & $0.50 \%$ & 38.00 & & & 38.00 \\
\hline Invalidez y vida & $1.75 \%$ & 133.00 & $0.63 \%$ & 47.50 & 180.50 \\
\hline Guarderías y prestaciones sociales & $1 \%$ & 76.00 & & & 76.00 \\
\hline Cesantía y vejez & $3.15 \%$ & 239.40 & $1.13 \%$ & 85.50 & 324.90 \\
\hline SUMAS IMSS & & 1082.69 & & 184.26 & 1266.95 \\
\hline Retiro & $2 \%$ & 152.00 & & & 152.00 \\
\hline INFONAVIT & $5 \%$ & 380.00 & & & 380.00 \\
\hline Sumas RCV e INFONAVIT & & 532.00 & & 0.00 & 532.00 \\
\hline Totales & & $1,614.69$ & & $\$ 184.26$ & $1,798.95$ \\
\hline
\end{tabular}

Nota: cálculo expresado en pesos mexicanos.

Fuente: elaboración propia de acuerdo con la ley del IMSS, INFONAVIT y RCV. 
Tabla 2. Cálculo de las COP con salario disminuido

\begin{tabular}{|c|c|c|c|c|c|}
\hline \multicolumn{6}{|l|}{ Cálculo salario real percibido } \\
\hline Salario percibido & & $\$ 95.54$ & & & \\
\hline Factor de integración & & 1.0466 & & & \\
\hline SDI & & $\$ 100$ & & & \\
\hline SBC & & $\$ 3,040$ & & & \\
\hline Años de antigüedad & & 2 & & & \\
\hline Cálculo & & Mensual & & & \\
\hline SMDF 2016 & & 73.04 & & & \\
\hline Pago IMSS & \multicolumn{2}{|c|}{ Patrón } & \multirow{2}{*}{\multicolumn{2}{|c|}{ Trabajador }} & Total \\
\hline Enfermedades y maternidad C. F & $20.40 \%$ & 452.96 & & & 452.96 \\
\hline Enfermedades y maternidad Excedente & $1.10 \%$ & 0.00 & $0.40 \%$ & 0.00 & 0.00 \\
\hline Enfermedades y maternidad esp. & $0.70 \%$ & 53.20 & $0.25 \%$ & 7.60 & 60.80 \\
\hline E y M esp. Pensionados & $1.05 \%$ & 31.92 & $0.38 \%$ & 11.40 & 43.32 \\
\hline Riesgo de trabajo & $0.50 \%$ & 15.20 & & & 15.20 \\
\hline Invalidez y vida & $1.75 \%$ & 53.20 & $0.63 \%$ & 19.00 & 72.20 \\
\hline Guarderías y prestaciones sociales & $1 \%$ & 30.40 & & & 30.40 \\
\hline Cesantía y vejez & $3.15 \%$ & 95.76 & $1.13 \%$ & 34.20 & 129.96 \\
\hline SUMAS IMSS & & 732.64 & & 72.20 & 804.84 \\
\hline Retiro & $2 \%$ & 60.80 & & & 60.80 \\
\hline INFONAVIT & $5 \%$ & 152.00 & & & 152.00 \\
\hline Sumas RCV e INFONAVIT & & 212.80 & & 0.00 & 212.80 \\
\hline Totales & & 945.44 & & $\$ 72.20$ & $1,017.64$ \\
\hline
\end{tabular}

Nota: cálculo expresado en pesos mexicanos.

Fuente: elaboración propia de acuerdo con la ley del IMSS, INFONAVIT y RCV.

Tabla 3. Resumen de afectación

\begin{tabular}{ccc}
\hline Institución & Diferencia & $\%$ \\
\hline IMSS & $\$ 462.11$ & $59.14 \%$ \\
INFONAVIT & $\$ 228.00$ & $29.19 \%$ \\
RETIRO & $\$ 91.20$ & $11.67 \%$ \\
\hline TOTAL & $\$ 781.31$ & $\mathbf{1 0 0 . 0 0 \%}$ \\
\hline
\end{tabular}

Nota: cálculo expresado en pesos mexicanos.

Fuente: elaboración propia con base en diferencia entre la Tabla 1 y la 2.

En la Tabla 3 se observa la afectación a las diversas instituciones a causa de la evasión de las COP. La más afectada es el IMSS con un $59.14 \%$, lo cual confirma que el ingreso es muy limitado si se compara con los servicios que se otorgan, razón por la que el IMSS es rebasado por la capacidad de los derechohabientes.

A continuación se expone un caso de estudio, con el fin de visualizar los perjuicios que pueden sufrir las empresas que no den de alta a sus trabajadores 
a tiempo, pues estos quedan en estado de indefensión al tener un riesgo de trabajo, y al patrón se le obliga a formar un capital constitutivo.

Se trata de un patrón, persona física, dedicado a la demolición de piedra, cuyo trabajador, que no contaba con seguridad social, cae en una trituradora y se fractura el fémur; por sus lesiones se genera amputación de la pierna izquierda, avulsión testicular bilateral y un choque hipovolémico.
El accidente de trabajo fue el 4 de mayo de 2010, la primera atención se realizó en el hospital central, que pertenece a la Secretaría de Salud. La atención por parte del IMSS fue el 18 de mayo de 2010. El patrón le dio el alta el 5 de mayo de 2010 con fecha de retroactividad al 3 de mayo de 2010.

En la Tabla 4 se dan a conocer las atenciones médicas que el Instituto le brindó al trabajador.

Tabla 4. Prestaciones en especies otorgadas por el IMSS

\begin{tabular}{|c|c|c|c|c|}
\hline \multirow{2}{*}{$\begin{array}{l}\text { Fecha de accidente o } \\
\text { inicio de enfermedad }\end{array}$} & Mes & Año & \multirow{2}{*}{ Fecha de alta } & \multirow{2}{*}{$\begin{array}{c}\text { Día/mes/año } \\
\text { 14-May-21 }\end{array}$} \\
\hline & 5 & 2010 & & \\
\hline Concepto & $\begin{array}{l}\text { Nivel de atención } \\
\text { médica }\end{array}$ & $\begin{array}{l}\text { Cantidad } \\
\text { de servicios } \\
\text { prestados }\end{array}$ & Costo unitario & Importe \\
\hline Atención del servicio de urgencias & Segundo nivel & 1 & $\$ 1.144,00$ & $\$ 1.144,00$ \\
\hline Consulta de especialidades & Segundo nivel & 16 & $\$ 850,00$ & $\$ 13.600,00$ \\
\hline Hospitalización días paciente & Segundo nivel & 52 & $\$ 4.939,00$ & $\$ 256.828,00$ \\
\hline Intervención quirúrgicas & Segundo nivel & 10 & $\$ 15.396,00$ & $\$ 153.960,00$ \\
\hline Estudios de laboratorio clínico & Segundo nivel & 8 & $\$ 84,00$ & $\$ 672,00$ \\
\hline Estudio de radiodiagnóstico ( Rayos X) & Segundo nivel & 1 & $\$ 278,00$ & $\$ 278,00$ \\
\hline Consulta de medicina familiar & Primer nivel & 2 & $\$ 535,00$ & $\$ 1.070,00$ \\
\hline \multicolumn{5}{|l|}{ Fechas de atención medicas } \\
\hline Urgencias 18/05/2010 & H.G.Z. no 50 & & & \\
\hline $\begin{array}{c}\text { Ortopedia } 20 / 07 / 2010,28 / 07 / 2010,25 / \\
08 / 2010,20 / 09 / 2010,11 / 10 / 2010,05 / 11 \\
/ 2010,08 / 11 / 2010,30 / 11 / 2010,27 / 10 / 20 \\
10,23 / 01 / 2011,21 / 02 / 2011,07 / 03 / 2011\end{array}$ & H.G.Z. no 51 & & & \\
\hline $\begin{array}{c}\text { Urología } 11 / 08 / 2010,25 / 10 / 20 \\
10,13 / 12 / 2010,11 / 03 / 2011\end{array}$ & H.G.Z. no 52 & & & \\
\hline $\begin{array}{c}\text { Hospitalización del } \\
18 / 05 / 2010 \text { a 08/07/2010 }\end{array}$ & H.G.Z. no 53 & & & \\
\hline $\begin{array}{l}\text { Intervenciones quirúrgicas: } 20 / 05 / 20 \\
\text { 10,28/05/2010,03/06/2010,08/06/201 } \\
\text { 0,11/06/2010,18/06/2010,22/06/2010, } \\
\text { 22/06/2010,25/06/2010,01/07/2010. }\end{array}$ & H.G.Z. no 54 & & & \\
\hline
\end{tabular}


Continuación Tabla 4

\begin{tabular}{cccccc}
\hline $\begin{array}{c}\text { Fecha de accidente o } \\
\text { inicio de enfermedad }\end{array}$ & día & Mes & Año & \multirow{2}{*}{ Fecha de alta } & Día/mes/año \\
\cline { 2 - 3 } & 4 & 5 & 2010 & & \\
\hline $\begin{array}{c}\text { Estudios de laboratorio: } 19 / 05 / 2010,20 / \\
05 / 2010,21 / 10 / 2010,09 / 06 / 2010,10 / 06 / 2\end{array}$ & H.G.Z. no 55 & & \\
$010,22 / 06 / 2010,24 / 06 / 2010,26 / 10 / 2010$ & & & & \\
& & & & \\
\hline Rayos X: $18 / 05 / 2010$ & H.G.Z. no 56 & & \\
\hline $\begin{array}{c}\text { Medicina familiar : } \\
\text { 19/05/2010,16/12/2010 }\end{array}$ & H.G.Z. no 57 & & \\
\hline & Total & & & \\
\hline & & & & & \\
\hline
\end{tabular}

Fuente: Instituto Mexicano del Seguro Social (2017).

Para el cálculo de las incapacidades se determinó un SBC de $\$ 119.45$, que cubrían desde el 4 de mayo de 2010 al 13 de abril de 2011, es decir, 345 días de incapacidad y un total de $\$ 41210.25$.

Se determinó un $100 \%$ de incapacidad permanente total $\mathrm{y}$, de acuerdo con el artículo 58, Fracc. II, el valor de la pensión es del $70 \%$ de su salario, más un aguinaldo de 15 días, en total $\$ 31791.12$.
Se calculó un promedio de vida de 19 años y se determinó una pensión total de $\$ 601662.84$, además, por el $5 \%$ de gastos administrativos, la cantidad de $\$ 53$ 521.25.

Como resumen, en la Tabla 5 se muestra un concentrado del capital constitutivo aplicado.

Tabla 5. Concentrado del capital constitutivo

\begin{tabular}{cc}
\hline Conceptos & Importe \\
\hline Prestaciones en especie & $\$ 427.552,00$ \\
Subsidios & $\$ 41.210,25$ \\
Valor actual de la pensión & $\$ 601.662,84$ \\
Subtotal & $\$ 1.070 .425,09$ \\
$5 \%$ Gastos de administración & $\$ 53.521,25$ \\
Total a pagar & $\$ 1.123 .946,34$ \\
\hline
\end{tabular}

Fuente: Instituto Mexicano del Seguro Social (IMSS, 2017). 


\section{CONCLUSIONES}

Teniendo en cuenta los resultados presentados, en primera instancia se reflejan las afectaciones que se generan a las finanzas del IMSS a causa de las omisiones de las COP que los patrones, para ahorrar costos, presentan en forma disminuida; lo cual, como se observó en el ejemplo, afecta en un $60 \%$ los ingresos del IMSS, situación que se traduce en problemas financieros para cumplir sus obligaciones, pues se encuentra sobrepasado por los servicios solicitados por los derechohabientes. Los patronos, por su parte, corren el riesgo de ser multados e incluso ser privados de su libertad (art. 308 de la ley del IMSS) por defraudación fiscal. Los perjuicios para el trabajador se muestran en la aportación disminuida a su cesantía en edad avanzada o vejez, puesto que al momento de querer jubilarse no tendrá el ingreso que percibía realmente; en cuestión del INFONAVIT la aportación será menor para un crédito hipotecario y en lo relativo a prestaciones en dinero será proporcional al salario que tiene registrado en el IMSS y no el que realmente percibe, lo cual lo deja en estado de indefensión al momento de solicitarlas. Por ejemplo, en caso de un riesgo de trabajo que cause la muerte del trabajador, se otorga una pensión por viudez a su pareja del $40 \%$ que le hubiese correspondido al asegurado por una IPT. La viuda recibe por parte del IMSS $\$ 840.00$ como pensión, aunque, de acuerdo con el salario real, debía percibir \$2100.00; por consiguiente, el patrón está obligado a pagar a la viuda $\$ 1260.00$ mensuales, que representan el $60 \%$, lo que afecta las prestaciones de gratificación anual que debe percibir por la cantidad de $\$ 630.00$, es decir, en un año el patrón del trabajador fallecido debe otorgar \$15 750 por indemnización y aguinaldo.

En el caso de los resultados del accidente de trabajo de un trabajador que no se encuentra inscrito en el IMSS, se observa la cantidad que tiene que pagar el patrón cuando se forma un capital constitutivo, que incluye los servicios de atención médica y prestaciones en dinero, más los cargos administrativos, lo cual implica poner en riesgo las finanzas de las empresas. Y en esto coincidimos con Muñoz et al. (2014), pues un capital constitutivo en algunos casos pone en riesgo la capacidad financiera y operativa de la empresa, que se ve limitada para responder por sus obligaciones.

Se concluye que las afectaciones a instituciones, empresas y trabajadores pueden ser de gran magnitud debido a las malas prácticas de evasión de cuotas patronales, ya sea por disminución de salario o simplemente por no registrarlos. Por lo cual se recomienda, en primer lugar, a los encargados de tomar estas decisiones en las empresas, que evalúen el riesgo de incurrir en estas prácticas; en segundo lugar, a las instituciones encargadas de la vigilancia permanente de los patrones, que cumplan sus funciones de manera correcta y oportuna; 
y a los trabajadores, que denuncien este tipo de prácticas que los deja indefensos en el evento de un riesgo de trabajo o una incapacidad, además de afectar su ahorro para el retiro y para acceder a un crédito hipotecario.

\section{AGRADECIMIENTOS}

Se agradece al Instituto Mexicano del Seguro Social por compartir información fundamental para la elaboración del artículo; de igual manera a los evaluadores anónimos de la Revista.

\section{DECLARACIÓN DE CONFLICTO DE INTERESES}

Los autores declaran que no tienen ningún conflicto de intereses.

\section{CONTRIBUCIÓN DE LOS AUTORES}

Los autores trabajaron de manera conjunta durante la construcción de todo el manuscrito.

\section{FINANCIAMIENTO:}

Este trabajo se realizó con recursos propios. 


\section{REFERENCIAS}

[1] Banco Mundial. (2021, 25 de mayo). Población mundial. https://datos.bancomundial.org/indicator/SP.POP.TOTL

[2] Centro de Estudios de las Finanzas Públicas. (2013). Indicadores sobre seguridad social en México. Cámara de Diputados. http://archivos.diputados.gob.mx/ Centros_Estudio/Cesop/Comisiones/2_ssocial.htm

[3] Comisión Representativa ante Organismos de Seguridad Social. (2017). Estudio integral de las pensiones que otorga el IMSS. (2. ${ }^{\mathrm{a}}$ ed.). Instituto Mexicano de Contadores Públicos, A.C.

[4] Constitución Política de los Estados Unidos Mexicanos. (2019). Texto de la Constitución. Diario Oficial de la Federación.

[5] Cortés, J. A., Rincón, L., Rivera, Y. \& Soto, L. (2011). La evasión y elusión en el Sistema General de Riesgos Profesionales de las organizaciones afiliadas al Sistema General de Seguridad Social, en la ciudad de Bogotá, D.C., 2000-2006. Nova, 9(15), 138-148. http://dx.doi.org/10.22490/24629448.496

[6] Hernández-Sampieri, R. \& Mendoza, C. (2018). Metodología de la investigación: las rutas cuantitativas, cualitativas y mixtas. McGraw-Hill.

[7] Instituto Mexicano del Seguro Social -IMSS-. (2017). Archivo. México. http:// www.imss.gob.mx/prensa/archivo/201818/012\#text $=\mathrm{E} 1 \% 2019 \% 20 \mathrm{de} \% 20$ enero $\% 20$ de,de $\% 2010$ \% $\% 20$ trabajadores $\% 20$ al $\% 20$ desarrollo.ytext $=\mathrm{A} \% 2075 \% 20$ a $\%$ C3\%B1os\%20de\%20su,sus\%2074\%20millones\%20de\%20derechohabientes

[8] Instituto Mexicano del Seguro Social -IMSS-. (2021). Puestos de trabajo afiliados al Instituto Mexicano del Seguro Social. http://www.imss.gob.mx/prensa/ archivo/202010/691

[9] Levy, S. (2010), Good Intentions, Bad Outcomes. Social Policy, Informality and Economic Growth in México. Brookings Institution Press.

[10] Ley Federal del Trabajo. (2006). Cámara de diputados del H. Congreso de la Unión. México, Diario Oficial de la Federación.

[11] Medina, A. \& Flores, U. (2017). La seguridad social como derecho humano en el contexto internacional. Revista de Investigación en Derecho, Criminología y Consultoría Jurídica, 111-128. http://dx.doi.org/10.32399/rdk.10.20.239 
[12] Ministerio de Salud y Protección Social -Minsalud-. (2021). Régimen subsidiado. Colombia. https://www.minsalud.gov.co/proteccionsocial/Regimensubsidiado/ Paginas/coberturas-del-regimen-subsidiado.aspx

[13] Montekio, V., Medina, G. \& Aquino, R. (2011). Sistema de salud de Brasil. Salud Pública de México, 53(Supl. 2), 120-131. http://www.scielo.org.mx/scielo. php?Script=sci_arttext\&pid=S0036-36342011000800008\&lng=es\&tlng=es

[14] Muñoz, C., Ortigoza, A., Reyes, A. \& Fernández. S. (2014). Impacto financiero de un capital constitutivo en una pyme. Revista del Centro de Investigación Universidad de La Salle, 11(42), 87-103. https://www.redalyc.org/articulo. oa?id=342/34235376003

[15] Organización Internacional del Trabajo -OIT-. (2010). Hechos concretos sobre la seguridad social. https://www.ilo.org/global/resources/WCMS_067592/lang--es/ index.htm

[16] Organización Internacional del Trabajo -OIT-. (2021). Reportes. https://www. ilo.org/wcmsp5/groups/public/---dgreports/--dcomm/documents/publication/ wcms_067592.pdf

[17] Sáenz, M., Acosta, M. Muiser, J. \& Bermúdez. (2011). Sistema de salud de Costa Rica. Salud Pública de México, 53(Supl. 2), 156-167. http://www.scielo.org.mx/ scielo.php?Script=sci_arttext\&pid=S0036-36342011000800011\&lng=es\&tlng=es

[18] Servicio de Administración Tributaria. (2018). Informe tributario. México. http:// www2.sat.gob.mx/sitio_internet/informe_tributario/itg2014t2/glosario.pdf

[19] Subsecretaría de Previsión Social. (2021) Informe estadístico semestral de la seguridad social. Segundo semestre de 2020. Chile. https://www.previsionsocial. gob.cl/sps/download/estudios-previsionales/estadisticas-previsionales/informe-estadistico-semestral-de-seguridad-social/Segundo-Semestre_2020.pdf 